\title{
Effects of ulinastatin treatment on myocardial and renal injury in patients undergoing aortic valve replacement with cardiopulmonary bypass
}

\author{
Se-young $\mathrm{Oh}^{1}$, Jong Chan Kim², Yong Seon $\mathrm{Choi}^{1}$, Woo Kyung Lee ${ }^{1}$, Yeong-Kyu Lee ${ }^{1}$, and \\ Young Lan Kwak ${ }^{1}$
}

Department of Anesthesiology and Pain Medicine, ${ }^{1}$ Yonsei University College of Medicine, Seoul, ${ }^{2}$ Kyungpook National University Hospital, Daegu, Korea

Background: We determined the protective effects of a high dose of ulinastatin on myocardial and renal function in patients undergoing aortic valve replacement with cardiopulmonary bypass (CPB).

Methods: Sixty patients were assigned randomly to either the ulinastatin group $(n=30)$ or the control group $(n=30)$. In the ulinastatin group, ulinastatin $(300,000 \mathrm{U})$ was given after the induction of anesthesia, ulinastatin $(400,000 \mathrm{U})$ was added to the CPB pump prime, and then ulinastatin (300,000 U) was administered after weaning from CPB. In the control group, the same volume of saline was administered at the same time points. Creatine kinase-MB levels were assessed 1 day before surgery, and on the first and second postoperative day (POD 1 and 2). Serum creatinine and cystatin $C$ levels were assessed 1 day before surgery, upon intensive care unit arrival, and on POD 1 and 2. The level of plasma neutrophil gelatinase-associated lipocalin was assessed before induction of anesthesia, upon ICU arrival, and on POD 1.

Results: No significant differences were observed in serum levels of creatine kinase-MB and biomarkers of renal injury between the two groups at any point during the study period.

Conclusions: Ulinastatin showed no cardiac or renal protective effects after CPB in patients undergoing aortic valve replacement. (Korean J Anesthesiol 2012; 62: 148-153)

Key Words: Cardiac surgical procedures, Cardiopulmonary bypass, Myocardiac protection.

Received: July 15, 2011. Revised: 1st, August 15, 2011; 2nd, August 26, 2011. Accepted: August 29, 2011.

Corresponding author: Yong Seon Choi, M.D., Department of Anesthesiology and Pain Medicine, Yonsei University College of Medicine, 250, Seongsanno, Seodaemun-gu, Seoul 120-752, Korea. Tel: 82-2-2227-4576, Fax: 82-2-364-2951, E-mail: yschoi@yuhs.ac

(c) This is an open-access article distributed under the terms of the Creative Commons Attribution Non-Commercial License (http:// creativecommons.org/licenses/by-nc/3.0/), which permits unrestricted non-commercial use, distribution, and reproduction in any medium, provided the original work is properly cited. 


\section{Introduction}

Open heart surgery using cardiopulmonary bypass (CPB) is invariably accompanied by a systemic inflammatory response and multiple-organ ischemia/reperfusion (I/R) injury [1]. Myocardial dysfunction and acute renal injury after $\mathrm{CPB}$, which lead to a prolonged postoperative hospital stay, continue to be the subject of a considerable amount of clinical research. Although the mechanisms of CPB-associated myocardial and renal injury have yet to be fully elucidated, the release of protease enzymes, cytokines, and oxygen free radicals from activated neutrophils results in endothelial injury, platelet activation, and a sequential inflammatory cascade, leading to post-pump organ injury [2-6].

Ulinastatin, a urinary trypsin inhibitor, is a nonspecific protease inhibitor and glycoprotein that is extracted and purified from human urine. It suppresses inflammatory activity, infiltration of neutrophils, and release of elastase and chemical mediators from neutrophils [7-12]. In addition, ulinastatin protects mitochondrial function by directly inhibiting lysosomal enzymes and oxygen free radicals in cells and by reducing calcium overload in injured cells [13]. Ulinastatin may reduce post-pump myocardial and renal injury by suppressing the release of proinflammatory cytokines and neutrophil elastase. Although some studies have reported that ulinastatin has a beneficial effect on I/R injury of the heart and kidneys, the sample sizes of these studies were too small to determine clinical outcome after open heart surgery under CPB [12,14]. Additionally, little is known about whether a high dose of ulinastatin $(1,000,000 \mathrm{U})$ protects the heart and kidneys from I/ R injury in adult patients undergoing valvular heart surgery.

Therefore, we performed a prospective, randomized, doubleblind, placebo-controlled clinical study with a homogeneous group of patients undergoing aortic valve replacement. Our aim was to evaluate whether the administration of high dose ulinastatin could provide myocardial and renal protection during CPB.

\section{Materials and Methods}

After approval of this study protocol by our Institutional Review Board, we obtained informed consent from 60 adult patients scheduled for elective aortic valve replacement between February 2009 and March 2011. We excluded patients who underwent combined valve and coronary-artery bypass grafting procedures, those with active infective endocarditis, hepatic or pulmonary disease, left ventricular ejection fraction $<30 \%$, pre-existing renal dysfunction (serum creatinine level $>1.6 \mathrm{mg} / \mathrm{dl}$ for men and $1.4 \mathrm{mg} / \mathrm{dl}$ for women), those older than 80 years, and those who had undergone reoperations.
All preoperative cardiac medications, except diuretics, were continued until the day of surgery. All patients received standardized anesthetic management. Standard monitoring devices were used in the operating room. Anesthesia was induced with intravenous midazolam $(0.03-0.07 \mathrm{mg} / \mathrm{kg})$, sufentanil (1.5-3.0 $\mu \mathrm{g} / \mathrm{kg})$, and $0.9 \mathrm{mg} / \mathrm{kg}$ rocuronium bromide, and maintained with sevoflurane $(0.8-1.5 \%)$ and continuous infusion of sufentanil $(0.5-1.5 \mu \mathrm{g} / \mathrm{kg} / \mathrm{hr})$. After intubation, the patient's lungs were ventilated with a tidal volume of $8 \mathrm{ml} /$ $\mathrm{kg}$, an inspiratory/expiratory time ratio of $1: 2$, and a positive end-expiratory pressure of $5 \mathrm{~cm} \mathrm{H}_{2} \mathrm{O}$ in $40 \%$ oxygen with air. Respiratory rate was adjusted to between 8 and 12 breaths/min to maintain normocarbia.

After induction of anesthesia, patients were assigned randomly to either the ulinastatin group $(n=30)$ or the control group $(n=30)$, using a computerized randomization table. An anesthesia research nurse prepared the syringes of blinded solution that were administered by the attending anesthesiologist. In the ulinastatin group, patients received $300,000 \mathrm{U}$ ulinastatin intravenously in $50 \mathrm{ml}$ normal saline for $15 \mathrm{~min}$ after the induction of anesthesia with 400,000 $\mathrm{U}$ added to the CPB pump prime, followed by an intravenous infusion of 300,000 U ulinastatin in $50 \mathrm{ml}$ normal saline for $15 \mathrm{~min}$ after weaning from CPB. Patients received similar volumes of normal saline in the control group. All physicians caring for patients were blinded to the treatment allocation.

All patients received standardized surgical and CPB management. CPB was instituted with a membrane oxygenator primed with $1,200 \mathrm{ml}$ of crystalloid solution, $200 \mathrm{ml}$ of $20 \%$ mannitol, and $100 \mathrm{ml}$ of $20 \%$ albumin. Surgical procedures were performed under mild hypothermia $\left(32-33^{\circ} \mathrm{C}\right)$. Acid-base management was performed with the $\alpha$-stat method, and the target range for $\mathrm{PaO}_{2}$ was $200-300 \mathrm{mmHg}$. The non-pulsatile pump flow rate was maintained at $2.0-2.5 \mathrm{~L} / \mathrm{min} / \mathrm{m}^{2}$, and a blood cardioplegic solution was used. Patients were weaned from CPB after completion of the surgical procedure and systemic re-warming. During the perioperative period and CPB, mean arterial pressure was maintained at $60-80 \mathrm{mmHg}$. Norepinephrine was used for vasopressor support and milrinone for inotropic support. The threshold for transfusion of packed red blood cells was a hematocrit $<20 \%$ during $\mathrm{CPB}$ and $25 \%$ after CPB. The blood from the CPB circuit was salvaged (cell salvage device) after heparin was reversed and retransfused after sternal closure. Urine output and all fluids administered to patients were recorded until the first postoperative day (POD1).

All patients were transferred to the intensive care unit (ICU) after surgery, where they received management according to institutional guidelines. Fluid therapy was administered postoperatively to maintain pulmonary capillary wedge pressure at $10-14 \mathrm{mmHg}$, cardiac index $>2 \mathrm{~L} / \mathrm{min} / \mathrm{m}^{2}$, and 
urine output $>0.5 \mathrm{ml} / \mathrm{kg} / \mathrm{h}$. The duration of ventilation, length of ICU stay, and postoperative hospitalization length of stay were recorded.

Hemodynamic variables, including the cardiac index and the number of patients requiring norepinephrine, were recorded $15 \mathrm{~min}$ after tracheal intubation, $15 \mathrm{~min}$ after sternal closure, and 1 and 24 hours after ICU arrival. Serum creatinine and cystatin $C$ levels were assessed 1 day before surgery, upon ICU arrival, and on POD 1 and the second postoperative day (POD 2). Blood samples were collected to measure levels of plasma neutrophil gelatinase-associated lipocalin (NGAL) before induction of anesthesia, upon ICU arrival, and on POD 1. Serum was separated from blood by centrifugation and stored at $-70^{\circ} \mathrm{C}$ until assay. Plasma NGAL levels were measured using a commercially available ELISA kit (Human Lipocalin-2/ NGAL Immunoassay R\&D Systems, Minneapolis, MN, USA), according to the manufacturer's instructions. Creatine kinase-

Table 1. Patient Characteristics and Operative Data

\begin{tabular}{lccc}
\hline \multicolumn{1}{c}{ Variable } & $\begin{array}{c}\text { Control } \\
\text { group } \\
(\mathrm{n}=30)\end{array}$ & $\begin{array}{c}\text { Ulinastatin } \\
\text { group } \\
(\mathrm{n}=30)\end{array}$ & P value \\
\hline Age (yr) & $60 \pm 12$ & $67 \pm 19$ & 0.09 \\
Gender (M/F) & $16 / 14$ & $20 / 10$ & 0.43 \\
Body surface area $\left(\mathrm{m}^{2}\right)$ & $1.6 \pm 0.2$ & $1.7 \pm 0.2$ & 0.12 \\
Diabetes & $3(10)$ & $3(10)$ & 1.00 \\
Hypertension & $7(23)$ & $13(43)$ & 0.10 \\
Atrial fibrillation & 0 & $1(3)$ & 0.49 \\
LVEF (\%) & $62 \pm 14$ & $63 \pm 11$ & 0.97 \\
Pre-operative medications & & & \\
$\quad$ ACE-I/ARB & $12(40)$ & $6(20)$ & 0.16 \\
Calcium channel blocker & $5(17)$ & $7(23)$ & 0.53 \\
$\quad \beta$-blockers & $4(13)$ & $3(10)$ & 1.00 \\
$\quad$ Diuretics & $15(50)$ & $8(27)$ & 0.11 \\
NYHA class III or IV & $4(13)$ & $4(13)$ & 1.00 \\
Duration of ACC (min) & $76 \pm 21$ & $74 \pm 19$ & 0.73 \\
Duration of CPB (min) & $96 \pm 22$ & $99 \pm 25$ & 0.64 \\
\hline
\end{tabular}

Values are mean \pm SD or number of patients (\%). LVEF: left ventricular ejection fraction, ACE-I: angiotensin converting enzyme inhibitor, ARB: angiotensin receptor blocker, NYHA: New York Heart Association, ACC: aorta cross clamp, CPB: cardiopulmonary bypass.
MB (CK-MB) levels were assessed 1 day before surgery and on POD 1 and 2.

The primary endpoint was to compare post-pump myocardial enzyme leakage between the ulinastatin and control groups using the levels of serum CK-MB and serum biomarkers of renal injury, including serum creatinine, cystatin C, and NGAL.

Based on data from a previous study, the mean and standard deviation of postoperative CK-MB level, which is an index of myocardial injury, is $40 \pm 15 \mu \mathrm{g} / \mathrm{L}$ in patients undergoing aortic valve replacement [15]. We accepted that a $30 \%$ reduction in CK-MB level represented a clinically significant benefit. We estimated that a sample of 26 patients per group would be sufficient to detect a mean difference between groups with a power of 0.8 and a type I error of 0.05 . Factoring in a drop-out rate of $\sim 10 \%$, we calculated that 30 patients would be required for each group.

Data were analyzed with SPSS 18.0 (SPSS Inc., Chicago, IL, USA) and expressed as mean \pm SD or number of patients. Data between the groups were compared using the chi-square test, Fisher's exact test, independent t-test, or Mann-Whitney $U$-test, as appropriate. Repeated-measures analysis of variance followed by a post-hoc Dunnett's test was used for intragroup comparisons of variables compared to baseline values. A $\mathrm{P}$ value $<0.05$ was considered statistically significant.

\section{Results}

Patients in the two groups had similar characteristics. The mean duration of CPB and aortic cross-clamp were not different between the groups (Table 1). No significant differences in serum levels of CK-MB, creatinine, cystatin C, or NGAL were observed between the groups throughout the study period (Table 2).

The total volume of fluid infused and urine output were similar between the two groups during the operation and the first $24 \mathrm{hr}$ in the ICU. The total amount of pRBCs transfused between the groups was similar during the operation and for the first $48 \mathrm{hr}$ in the ICU (Table 3).

Table 2. Changes in Preoperative and Postoperative Cardiac and Renal Biomarkers

\begin{tabular}{|c|c|c|c|c|c|}
\hline Variables & Group & Baseline & ICU arrival & POD 1 & POD 2 \\
\hline \multirow[t]{2}{*}{ CK-MB (ng/ml) } & Control & $4.2 \pm 1.8$ & & $26.1 \pm 13.9$ & $18.5 \pm 9.7$ \\
\hline & Ulinastatin & $2.7 \pm 0.9$ & & $22.5 \pm 9.2$ & $16.4 \pm 7.1$ \\
\hline \multirow{2}{*}{ Creatinine (mg/dl) } & Control & $1.0 \pm 0.2$ & $0.9 \pm 0.2$ & $1.0 \pm 0.3$ & $1.0 \pm 0.2$ \\
\hline & Ulinastatin & $1.0 \pm 0.2$ & $0.9 \pm 0.1$ & $1.1 \pm 0.2$ & $1.0 \pm 0.2$ \\
\hline \multirow[t]{2}{*}{ Cystatin C (mg/L) } & Control & $1.0 \pm 0.2$ & $0.8 \pm 0.2$ & $0.9 \pm 0.3$ & $1.0 \pm 0.3$ \\
\hline & Ulinastatin & $0.9 \pm 0.2$ & $0.8 \pm 0.2$ & $0.9 \pm 0.3$ & $1.2 \pm 0.3$ \\
\hline \multirow[t]{2}{*}{ Plasma NGAL (ng/ml) } & Control & $41 \pm 21$ & $149 \pm 58$ & $144 \pm 51$ & \\
\hline & Ulinastatin & $57 \pm 51$ & $151 \pm 57$ & $148 \pm 57$ & \\
\hline
\end{tabular}

Values are mean \pm SD or number of patients. ICU: intensive care unit, POD: postoperative day, CK-MB: creatine kinase-MB, NGAL: neutrophil gelatinase-associated lipocalin. 
Hemodynamic variables, including the cardiac index, were not significantly different between the groups throughout the study period (Table 4). The total amount of norepinephrine used during weaning from CPB did not differ between the control group and the ulinastatin group $(200 \pm 158 \mu \mathrm{g}$ vs. $230 \pm$ $158 \mu \mathrm{g}, \mathrm{P}=0.471)$.

The time to extubation, postoperative length of stay in the ICU, and postoperative hospitalization period were also similar between the two groups (Table 5).

\section{Discussion}

In this prospective, randomized trial designed to investigate the myocardial and renal protective effects of a high dose of ulinastatin in patients undergoing aortic valve replacement, we did not observe any beneficial effects of ulinastatin, as assessed by monitoring serum biomarkers of myocardial and renal injury.

Table 3. Perioperative Fluid Balance and Transfusion Data

\begin{tabular}{lccc}
\hline \multicolumn{1}{c}{ Variable } & $\begin{array}{c}\text { Control } \\
\text { group } \\
(\mathrm{n}=30)\end{array}$ & $\begin{array}{c}\text { Ulinastatin } \\
\text { group } \\
(\mathrm{n}=30)\end{array}$ & P value \\
\hline Fluid input (ml) & & & \\
$\quad$ OR & $1,958 \pm 545$ & $2,200 \pm 676$ & 0.23 \\
ICU 24 hr & $4,599 \pm 667$ & $4,342 \pm 886$ & 0.14 \\
Urine output (ml) & $1,239 \pm 1,713$ & $986 \pm 427$ & 0.42 \\
OR & $3,299 \pm 651$ & $3,231 \pm 852$ & 0.47 \\
ICU 24 hr & $338 \pm 198$ & $394 \pm 220$ & 0.57 \\
Blood loss during ICU 24 hr (ml) & & & \\
pRBC transfused (units) & $0.8 \pm 0.9$ & $0.6 \pm 0.8$ & 0.55 \\
OR & $0.2 \pm 0.6$ & $0.6 \pm 0.9$ & 0.17 \\
ICU 24 hr & $0.1 \pm 0.5$ & $0.2 \pm 0.6$ & 0.74 \\
ICU 24-48 hr & & & \\
\hline
\end{tabular}

Values are mean \pm SD. OR: operating room, ICU: intensive care unit, pRBC: packed red blood cells.
Ulinastatin is a complex polypeptide extracted from human urine that suppresses the release of inflammatory chemical mediators such as polymorphonuclear neutrophil elastase (PMNE), tumor necrosis factor-alpha, interleukin (IL)-6, and IL8. Several studies have suggested that ulinastatin can reduce $I / R$ injuries of major organs such as the kidneys, lungs, and liver [4,9$12,16]$. While ulinastatin was revealed to have a protective effect on myocardial injury in animal experiments $(15,000 \mathrm{U} / \mathrm{kg})$ and pediatric patients $(12,000 \mathrm{U} / \mathrm{kg})[14,17]$, adult cardiac surgery studies $(5,000-6,000 \mathrm{U} / \mathrm{kg})$ have reported conflicting results $[18,19]$. In these studies, doses of ulinastatin administered to adult patients were relatively small compared to those used in animal experiments and in pediatric studies [14,17-19]. However, patients who underwent coronary-artery bypass graft surgery (CABG) with $\mathrm{CPB}$ and who received 1,000,000 $\mathrm{U}$ ulinastatin have lower levels of PMNE and inflammatory cytokines than those in a control group, and their weaning time from mechanical ventilation is shortened [4]. Therefore, we performed this clinical study to evaluate the impact of a high dose of ulinastatin $(1,000,000 \mathrm{U})$ on myocardial and renal injury in a homogeneous group of patients. However, we did not observe myocardial and renal protection effects in adult

Table 5. Postoperative Outcome Data

\begin{tabular}{lccc}
\hline \multicolumn{1}{c}{ Variable } & $\begin{array}{c}\text { Control } \\
\text { group } \\
(\mathrm{n}=30)\end{array}$ & $\begin{array}{c}\text { Ulinastatin } \\
\text { group } \\
(\mathrm{n}=30)\end{array}$ & $\begin{array}{c}\mathrm{P} \\
\text { value }\end{array}$ \\
\hline Reintubation & 0 & 1 & 1.00 \\
Time on ventilator (hr) & $16.1 \pm 4.5$ & $16.7 \pm 4.8$ & 0.66 \\
Norepinephrine use & $6(20)$ & $9(30)$ & 0.70 \\
ICU stay (days) & $2.9 \pm 0.7$ & $3.0 \pm 0.4$ & 0.98 \\
Postoperative hospital stay (days) & $9.4 \pm 4.9$ & $10.4 \pm 6.2$ & 0.55 \\
\hline
\end{tabular}

Values are mean \pm SD or number of patients (\%). ICU: intensive care unit.

Table 4. Changes in Hemodynamic Variables at Various Time Points

\begin{tabular}{|c|c|c|c|c|c|}
\hline Variables & Group & $\mathrm{T} 1$ & $\mathrm{~T} 2$ & T3 & $\mathrm{T} 4$ \\
\hline \multirow[t]{2}{*}{ HR (beats/min) } & Control & $75 \pm 15$ & $80 \pm 12$ & $95 \pm 19$ & $89 \pm 7$ \\
\hline & Ulinastatin & $76 \pm 18$ & $83 \pm 11$ & $95 \pm 15$ & $87 \pm 11$ \\
\hline \multirow[t]{2}{*}{ MAP (mmHg) } & Control & $92 \pm 8$ & $67 \pm 9$ & $79 \pm 10$ & $75 \pm 10$ \\
\hline & Ulinastatin & $103 \pm 14$ & $67 \pm 13$ & $76 \pm 13$ & $76 \pm 8$ \\
\hline \multirow[t]{2}{*}{ MPAP (mmHg) } & Control & $20 \pm 7$ & $17 \pm 3$ & $21 \pm 23$ & $16 \pm 3$ \\
\hline & Ulinastatin & $21 \pm 6$ & $19 \pm 4$ & $15 \pm 4$ & $17 \pm 3$ \\
\hline \multirow[t]{2}{*}{ CVP (mmHg) } & Control & $7 \pm 3$ & $8 \pm 3$ & $7 \pm 3$ & $7 \pm 2$ \\
\hline & Ulinastatin & $7 \pm 3$ & $9 \pm 3$ & $8 \pm 2$ & $8 \pm 2$ \\
\hline \multirow[t]{2}{*}{ PCWP (mmHg) } & Control & $13 \pm 5$ & $12 \pm 2$ & $12 \pm 3$ & $12 \pm 3$ \\
\hline & Ulinastatin & $14 \pm 5$ & $14 \pm 4$ & $13 \pm 3$ & $13 \pm 3$ \\
\hline \multirow[t]{2}{*}{$\mathrm{CI}\left(\mathrm{L} / \mathrm{min} / \mathrm{m}^{2}\right)$} & Control & $2.9 \pm 0.8$ & $2.9 \pm 0.6$ & $3.4 \pm 0.9$ & $3.2 \pm 0.6$ \\
\hline & Ulinastatin & $3.1 \pm 1.0$ & $3.0 \pm 0.7$ & $3.1 \pm 0.6$ & $3.1 \pm 0.6$ \\
\hline
\end{tabular}

Values are mean \pm SD. T1: 15 min after tracheal intubation, T2: 15 min after sternum closure, T3 and T4: 1 and 24 hours after arrival at the intensive care unit, respectively, HR: heart rate, MAP: mean systemic arterial pressure, MPAP: mean pulmonary arterial pressure, CVP: central venous pressure, PCWP: pulmonary capillary wedge pressure, $\mathrm{CI}$ : cardiac index. 
patients who underwent aortic valve replacement after CPB.

The CK-MB values were not significantly different between the control and ulinastatin group during the postoperative period in this study. A previous study, which was performed in adult patients undergoing CABG or valvular surgery with $\mathrm{CPB}$, suggested that the administration of $10,000 \mathrm{U} / \mathrm{kg}$ ulinastatin has no influence on postoperative CK-MB level [20]. However, ulinastatin significantly reduces the postoperative increase in CK-MB levels at 24 hours after discontinuation of CPB in patients who underwent open heart surgery and received $12,000 \mathrm{U} / \mathrm{kg}$ ulinastatin [14]. Additionally, ulinastatin reduces the postoperative increase in troponin-I levels in patients who underwent off-pump coronary artery bypass graft surgery when it was infused initially at a concentration of $6,000 \mathrm{U} / \mathrm{kg}$ and then at a rate of $1,000 \mathrm{U} / \mathrm{kg} / \mathrm{hr}$ throughout the surgery [19]. This difference in outcomes between our study and these previous studies might be due to the degree of systemic inflammatory response, type of surgery, and the use of $\mathrm{CPB}$; we found that ulinastatin did not effectively exert a myocardial protective effect against I/R injury in patients who underwent aortic valve replacement using $\mathrm{CPB}$.

Acute kidney injury is evaluated mainly by measuring postoperative levels of creatinine, which is a low sensitivity technique. Therefore, we assessed whether ulinastatin had a renal protective effect by using the renal biomarkers cystatin $\mathrm{C}$ and NGAL, which predict kidney injury earlier and with more sensitivity than creatinine [21]. No significant differences in the postoperative levels of these three renal biomarkers were observed between the two groups. In previous studies reporting that ulinastatin has a renal protective effect after $\mathrm{CPB}$, the renal tubular enzyme, $\mathrm{N}$-acetyl-beta-D-glucosaminidase, which is collected from urine, was used, and significant differences in levels of this enzyme were observed between the control and ulinastatin group, but no significant differences were observed in creatinine levels between the two groups $[12,22]$. Ulinastatin has also been reported to prevent postoperative aggravation of renal function in patients with poor preoperative renal function [15]. In contrast, we observed no renal protective effect in this study. All patients except three patients in the control group and four patients in the ulinastatin group had well-functioning kidneys with a glomerular filtration rate $>60 \mathrm{ml} / \mathrm{min} / 1.73$ $\mathrm{m}^{2}$ preoperatively. Therefore, none of the patients in either group experienced acute kidney injury, defined as an absolute increase in serum creatinine levels by either $0.3 \mathrm{mg} / \mathrm{dl}$ or $\geq 50 \%$ from baseline [23].

Even though the main ulinastatin mechanism of action is to suppress the release of PMNE, a critical limitation of this study was that we were unable to check whether the administrated dose of ulinastatin adequately suppressed PMNE release. However, a previous study reported that a smaller dose of ulinastatin can inhibit PMNE release [24]. No differences were observed between the two groups when we compared the white blood cell and neutrophil counts to determine the degree of inflammation during the preoperative and postoperative periods.

There are several reasons why ulinastatin may not have had a myocardial and renal protective effect. First, although we used a high dose of ulinastatin compared with other studies, this bolus may not have been sufficient to suppress inflammatory reactions and activate PMNE, which are persistent during CPB. Furthermore, given that the plasma half-life of ulinastatin is short at about 40 minutes [25], injection of continuously infused high-dose ulinastatin would likely have been more effective. Second, the patients who were enrolled had a wellfunctioning heart and kidneys preoperatively; therefore, they were at low risk for organ injury after $\mathrm{CPB}$, because preoperative cardiac and renal function affects postoperative cardiac and renal function, respectively. We only included patients who had a normal, functioning heart and kidneys to allow us to objectively evaluate the effects of ulinastatin. A high dose of ulinastatin should be infused continuously in patients with poorly functioning kidneys to further investigate the effects of ulinastatin during heart surgery with CPB.

In conclusion, ulinastatin did not effectively reduce CBPinduced damage to the heart and kidneys of patients who underwent an aortic valve replacement.

\section{References}

1. Butler J, Rocker GM, Westaby S. Inflammatory response to cardiopulmonary bypass. Ann Thorac Surg 1993; 55: 552-9.

2. Suleiman MS, Zacharowski K, Angelini GD. Inflammatory response and cardioprotection during open-heart surgery: the importance of anaesthetics. Br J Pharmacol 2008; 153: 21-33.

3. Laffey JG, Boylan JF, Cheng DC. The systemic inflammatory response to cardiac surgery: implications for the anesthesiologist. Anesthesiology 2002; 97: 215-52.

4. Bingyang J, Jinping L, Mingzheng L, Guyan W, Zhengyi F. Effects of urinary protease inhibitor on inflammatory response during on-pump coronary revascularisation. Effect of ulinastatin on inflammatory response. J Cardiovasc Surg (Torino) 2007; 48: 497503.

5. Hansen PR. Role of neutrophils in myocardial ischemia and reperfusion. Circulation 1995; 91: 1872-85.

6. Nishiyama T, Yokoyama T, Yamashita K. Effects of a protease inhibitor, ulinastatin, on coagulation and fibrinolysis in abdominal surgery. J Anesth 2006; 20: 179-82.

7. Aosasa S, Ono S, Mochizuki H, Tsujimoto H, Ueno C, Matsumoto A. Mechanism of the inhibitory effect of protease inhibitor on tumor necrosis factor alpha production of monocytes. Shock 2001; 15: 101-5.

8. Sato Y, Ishikawa S, Otaki A, Takahashi T, Hasegawa Y, Suzuki M, 
et al. Induction of acute-phase reactive substances during openheart surgery and efficacy of ulinastatin. Inhibiting cytokines and postoperative organ injury. Jpn J Thorac Cardiovasc Surg 2000; 48: 428-34.

9. Aihara T, Shiraishi M, Hiroyasu S, Hatsuse K, Mochizuki H, Seki S, et al. Ulinastatin, a protease inhibitor, attenuates hepatic ischemia/ reperfusion injury by downregulating TNF-alpha in the liver. Transplant Proc 1998; 30: 3732-4.

10. Nakahama H, Obata K, Sugita M. Ulinastatin amelirates acute ischemic renal injury in rats. Ren Fail 1996; 18: 893-8.

11. Sugita T, Watarida S, Katsuyama K, Nakajima Y, Yamamoto R, Mori A. Effect of a human urinary protease inhibitor (Ulinastatin) on respiratory function in pediatric patients undergoing cardiopulmonary bypass. J Cardiovasc Surg (Torino) 2002; 43: 437-40.

12. Ueki M, Yokono S, Nogaya J, Taie S, Komatsu H, Nakano M, et al. Effects of ulinastatin on postoperative renal function after cardiopulmonary bypass. Masui 1995; 44: 691-7.

13. Kanayama N, Halim A, Maehara K, Kajiwara Y, Fujie M, Terao T. Kunitz-type trypsin inhibitor prevents LPS-induced increase of cytosolic free $\mathrm{Ca} 2+$ in human neutrophils and HUVEC cells. Biochem Biophys Res Commun 1995; 207: 324-30.

14. Ren TY, Yang XW, Ma Y, Wang F, Qiang WD. Myocardial protective effect of ulinastatin against ischemia/reperfusion injury during open heart surgery with cardiopulmonary bypass. Zhonghua Yi Xue Za Zhi 2003; 83: 1391-3.

15. Braathen B, Vengen OA, Tønnessen T. Myocardial cooling with ice-slush provides no cardioprotective effects in aortic valve replacement. Scand Cardiovasc J 2006; 40: 368-73.

16. Yu XY, Fan LL. Effects of different doses of ulinastatin on inflammatory response and pulmonary function after cardiopulmonary bypass. Zhongguo Wei Zhong Bing Ji Jiu Yi Xue 2009; 21: 664-7.

17. Cao ZL, Okazaki Y, Naito K, Ueno T, Natsuaki M, Itoh T. Ulinastatin attenuates reperfusion injury in the isolate blood-perfused rabbit heart. Ann Thorac Surg 2000; 69: 1121-6.

18. Nakanishi K, Takeda S, Sakamoto A, Kitamura A. Effects of ulinastatin treatment on the cardiopulmonary bypass-induced hemodynamic instability and pulmonary dysfunction. Crit Care Med 2006; 34: 1351-7.

19. Wang GY, Qiu HB, Zhan SG, Li LH. Protection of ulinastatin against myocardial injury induced by off-pump coronary artery bypass graft surgery: report of 24 cases. Zhonghua Yi Xue Za Zhi 2007; 87: 2502-4.

20. Kawai T, Wada Y, Nishiyama K, Kanki Y, Ohga K, Oka T, et al. Usefulness of ulinastatin as a radical scavenger for protection of reperfusion injury after myocardial ischemia in open heart surgery. Nippon Kyobu Geka Gakkai Zasshi 1991; 39: 2157-62.

21. Haase-Fielitz A, Bellomo R, Devarajan P, Story D, Matalanis G, Dragun D, et al. Novel and conventional serum biomarkers predicting acute kidney injury in adult cardiac surgery--a prospective cohort study. Crit Care Med 2009; 37: 553-60.

22. Hashimoto K, Nomura K, Nakano M, Sasaki T, Kurosawa H. Pharmacological intervention for renal protection during cardiopulmonary bypass. Heart Vessels 1993; 8: 203-10.

23. Molitoris BA, Levin A, Warnock DG, Joannidis M, Mehta RL, Kellum JA, et al. Improving outcome of acute kidney injury: Report of an initiative. Nat Clin Pract Nephrol 2007; 3: 439-42.

24. Mishima A, Takiuti Y, Usami S, Ktani H, Suzuki Y, Yura J. Effects of ulinastatin on plasma polymorphonuclear leukocyte elastase activity and respiratory function in patients undergoing cardiopulmonary bypass. Nippon Kyobu Geka Gakkai Zasshi 1990; 38: 607-12.

25. Mitsuhata H, Enzan K, Hasegawa J, Matsumoto S, Yabe M, Matsumoto J. Effects of ulinastatin on changes of plasma granulocyte elastase, myeloperoxidase and fibronectin during and after open heart surgery. Masui 1990; 39: 1164-71. 